ACTA

ONCOLOGICA

mamuenrs

$=$

50
Acta Oncologica

ISSN: 0284-186X (Print) 1651-226X (Online) Journal homepage: https://www.tandfonline.com/loi/ionc20

\title{
Daily dose to organs at risk predicts acute toxicity in pancreatic stereotactic radiotherapy
}

\author{
Mauro Loi, Alba Magallon-Baro, Mustafa Suker, Casper Van Eijck, Mischa \\ Hoogeman \& Joost J. Nuyttens
}

To cite this article: Mauro Loi, Alba Magallon-Baro, Mustafa Suker, Casper Van Eijck, Mischa Hoogeman \& Joost J. Nuyttens (2020): Daily dose to organs at risk predicts acute toxicity in pancreatic stereotactic radiotherapy, Acta Oncologica, DOI: 10.1080/0284186X.2020.1742931

To link to this article: https://doi.org/10.1080/0284186X.2020.1742931

View supplementary material $\widetilde{\nearrow}$

曲 Published online: 24 Mar 2020.

Submit your article to this journal

Џll Article views: 10

Q View related articles $₫$

View Crossmark data $\asymp$ 


\title{
Daily dose to organs at risk predicts acute toxicity in pancreatic stereotactic radiotherapy
}

\author{
Mauro Loi ${ }^{\mathrm{a}}$, Alba Magallon-Baro ${ }^{\mathrm{a}}$, Mustafa Suker ${ }^{\mathrm{b}}$, Casper Van Eijck ${ }^{\mathrm{b}}$, Mischa Hoogeman ${ }^{\mathrm{a}}$ and Joost J. Nuyttens ${ }^{\mathrm{a}}$ \\ ${ }^{a}$ Department of Radiotherapy, Erasmus MC University Medical Centre, Rotterdam, The Netherlands; ${ }^{b}$ Department of Surgery, Erasmus MC \\ University Medical Centre, Rotterdam, The Netherlands
}

\section{Introduction}

Pancreatic adenocarcinoma (PC) is diagnosed as a locally advanced or borderline resectable disease in up to $40 \%$ of patients [1]. In nonmetastatic patients who are not eligible for upfront surgical treatment, multiple-agent chemotherapy is recommended [2] in order to select candidates for treatment intensification with radiotherapy as exclusive local treatment or as a neoadjuvant treatment before surgery [3-5]. In recent years, stereotactic body radiotherapy (SBRT) emerged as a promising alternative to conventional chemoradiation [6]. Results from phase $1 / / \mathrm{ll}$ trials [7] reported a cumulative local control rate of $71 \%$ at 1 year, and benefit of SBRT is currently evaluated in prospective phase III trials [8]. Moreover, irradiation up to $50 \mathrm{~Gy}$ in five fractions to part of the tumor has been correlated with an increase in local control and Ro resection rate, suggesting a potential interest of dose escalation to improve global outcome and disease downstaging in order to achieve surgical resectability $[9,10]$. However, development of more aggressive treatment schedules is limited by proximity of critical dose-limiting structures (duodenum, stomach, bowel), often resulting in a stringent tradeoff between adequate coverage of the target volume and preservation of dose constraints of the organs at risks (OARs). It has been shown that large inter-fractional physiological modifications in shape and position of OARs may occur, influencing daily dose distribution [11]. Determination of OAR motion on daily basis may be useful to improve critical structures protection while safely increasing dose to the target $[12,13]$. The aim of our study was to evaluate the performance of daily anatomical assessment to predict acute toxicity from abdominal SBRT in a prospective cohort of patients with locally advanced PC.

\section{Material and methods}

\section{Study population and treatment protocol}

Patients with locally advanced nonmetastatic PC were included in a prospective phase II single arm study testing SBRT following response or stable disease after eight cycles of FOLFIRINOX chemotherapy. The study was conducted according to the principles of the Declaration of Helsinki and was approved by Institutional Review Board with the number NL49643.078.14. Informed consent to the study procedures was signed by the patients. Before treatment initiation, fiducial markers were inserted in the tumor via endoscopic ultrasonography guidance. Gross tumor volume (GTV) was contoured on a $1.5-\mathrm{mm}$ thick contrast-enhanced CT scan. Clinical target volume (CTV) included the GTV plus geometric expansion of $5 \mathrm{~mm}$ to include potential microscopic tumor extension. Planning target volume (PTV) encompassed the CTV plus a $2 \mathrm{~mm}$ margin. Prescription dose to the $80 \%$ isodose line of the PTV was $40 \mathrm{~Gy}$ in $8 \mathrm{~Gy}$ daily fractions. At least $95 \%$ of the prescribed dose should cover $95 \%$ of the PTV, though PTV could be underdosed to meet the constraints of dose-limiting OARs. In particular, dose constraint for stomach, duodenum and bowel consisted of a maximum point dose (Dmax) of $35 \mathrm{~Gy}$, while spinal cord and liver were allowed to receive a Dmax of $27.5 \mathrm{~Gy}$ and less than $20 \mathrm{~Gy}$ to a volume inferior to $700 \mathrm{cc}$, respectively. Dose constraints are summarized in Supplementary Table 1a. Fiducial tracking was performed using the Synchrony respiratory motion tracking system. Clinical evaluation with physical examination and CT scan was performed at 1, 3 and 6 months, and subsequently once a year until 5 years after the treatment or until disease progression. According to study protocol, assessment of acute toxicity (occurring within 3 months from the first day of treatment) was prospectively performed at pre-specified time points ( 2 weeks, 1 month and 3 months) using the Common Terminology Criteria for Adverse Events (CTCAE V4.03, 2010).

\section{System description and evaluation of daily dose}

Detailed description of the system and clinical application for daily dose evaluation has been previously reported $[12,14]$. In summary, in our institution, a treatment platform integrating a CyberKnife with a CT scanner on-rail allows to perform daily imaging before irradiation. According to the LAPC-1 protocol, for the first three fractions of the treatment, an end-expiration CT scan with IV contrast was acquired in treatment position and was used for comparison. Daily CT 
scans were matched offline to the planning CT by applying a rigid registration based on spine match followed by a fiducials match correction, which was used to overlay the planned dose distribution on the daily CT after OAR delineation according to RTOG recommendations, as previously described $[12,14]$.

\section{Data analysis}

For each organ, the following metrics were extracted: volume receiving at least $35 \mathrm{~Gy}(\mathrm{~V} 35$, in $\mathrm{Cc})$, maximum dose received by $2 \mathrm{cc}(\mathrm{D} 2$, in Gy), maximum dose received by $5 \mathrm{cc}$ (D5, in Gy), maximum dose received by $10 \mathrm{cc}$ (D10, in Gy), and the maximum point dose to a single voxel (Dmax, in Gy). Three sets of value were collected, corresponding, respectively, to the parameter value as extracted from the simulation CTbased DVH ('planned'), to the average of each DVH parameter value after dose over the three daily CT scans ('daily') and to the highest value reached on one of the daily $C T$ scans ('peak'). For each OAR, correlation between planned, daily and peak values and specific acute toxicities (abdominal pain, nausea, diarrhea) was assessed using median comparison with the Kruskall-Wallis test. A receiver operating curve (ROC) was subsequently plotted to assess the performance of planned, daily and peak parameters to predict acute toxicity. An area under the curve (AUC) $>0.7$ indicated moderate-high accuracy. The optimal cutoff value in terms of sensitivity and specificity was obtained based on Youden-J index, corresponding to the dose or volume level for whom the maximum vertical distance between the ROC curve and the diagonal line was found [15].

\section{Results}

\section{Overview}

A prospective cohort of 39 patients underwent SBRT within the LAPC1 trial. Daily CT scans were not available for 4 patients due to logistics and therefore 35 patients (19 head and 16 body tumors) were included in the study. Median age was 61 years (range 48-75). Median tumor diameter was $38 \mathrm{~mm}$ (range 19-70), corresponding to a median GTV size of $26.8 \mathrm{cc}$ (range $5.1-141.0 \mathrm{cc}$ ): $31 \%$ of patients experienced a partial response following induction chemotherapy (See Supplementary Table $1 \mathrm{~b}$ for patient and tumor characteristics). All patients received the entire planned treatment course without interruption and were evaluated at 15 days, 1 and 3 months for toxicity according to study plan. Within 3 months from the beginning of the treatment, 22 (63\%) patients experienced acute treatment-related Grade 1-2 toxicity. This consisted of nausea in 11 patients, abdominal pain in 15 patients and diarrhea in 4 cases. Severity was mild, consisting of 12 Grade 1 and 18 Grade 2 toxicities. No Grade $\geq 3$ acute toxicity was observed (See Supplementary Table 1c).

\section{Acute nausea}

No difference was found at median comparison for dose parameters in stomach and bowel between patients with or without acute nausea (Supplementary Table 2). Conversely, dose distribution to duodenum was correlated to the onset of acute nausea: significantly higher median values were found in patients experiencing acute nausea compared to asymptomatic patients (Table 1 and Supplementary Table 2a). At ROC curve calculation, planned (D2, D5, D10, Dmax), daily (V35, D2, D5, D10) and peak (V35, D2, D5, D10) values showed an AUC $>0.7$ (Supplementary Figures 1 and 4). However, at a dose threshold of $32.2 \mathrm{~Gy}$, daily D2 yielded the highest accuracy ( $\mathrm{J}$ : 0.62) compared to its planned and peak counterpart (J: 0.57 and 0.48 , for 30.5 and 33.2 Gy respectively) (Figure 1(a) and Supplementary Table 3).

\section{Acute abdominal pain}

At median comparison, only dose parameters related to the stomach were associated with the presence of abdominal pain: in particular higher median planned (Dmax, D5, D10), daily (D2, D5, D10) and peak (D2, D5, D10) parameters were found significant (Table 1, Supplementary Table 2b). ROC curve calculation showed for each value an AUC $>0.7$ (Supplementary Table 3, Supplementary Figures 2 and 5). Comparable accuracy (J: 0.48) was found for planned and daily D10 at a dose threshold of 16.6 and $22.1 \mathrm{~Gy}$, respectively (Figure 1(b) and Supplementary Table 3).

\section{Acute diarrhea}

No differences were found between patients with or without treatment-related diarrhea at median comparison for dose parameters related to stomach and duodenum (Supplementary Table $2 a$ and b). Only median plan D10, daily D5, daily D10 and peak D10 to the bowels were significantly higher in patients with diarrhea compared to asymptomatic patients (Table 1 and Supplementary Table 2c). At ROC curve calculation, median plan D10, daily D5, daily D10 and peak D10 showed an AUC >0.8 (Supplementary Figures 3 and 6). Daily D10 yielded the highest accuracy (J: 0.71) at a threshold of 16.9 Gy (Figure 1(c) and Supplementary Table 3).

\section{Discussion}

Gastrointestinal acute toxicity is a frequent occurrence during radiation therapy of the upper abdomen, with important consequences in terms of global tolerability and compliance to treatment [16-18]. Most importantly, it may affect radiotherapy treatment planning, limiting the radiation dose that can be safely delivered [17]. Use of fractionated schedules reduced incidence of major toxicities from $42 \%$ in pivotal trials of single-fraction SBRT [19] to less than $10 \%$ in modern series using hypofractionated regimens [20,21]. In our experience, only minor acute G1-2 toxicities were observed, confirming that $40 \mathrm{~Gy}$ in 5 fractions is a feasible and welltolerated dose schedule. However, long-term follow-up is 
Table 1. Median comparison (Kruskall-Wallis Test) for selected parameters between patients with or without acute toxicity following SBRT.

\begin{tabular}{|c|c|c|c|c|}
\hline Duodenum & & Acute nausea (median [IQR]) & No acute nausea (median [IQR]) & $p$ \\
\hline \multirow[t]{5}{*}{ Planned } & V35 & $0.1[0.0-0.30] \mathrm{cc}$ & $0.0[0.0-0.20] c c$ & .07 \\
\hline & D2 & 32.6 [31.9-33.9] Gy & 26.7 [19.2-31.5] Gy & .021 \\
\hline & D5 & 30.5 [29.4-32.8] Gy & 21.4 [16.5-29.3] Gy & .021 \\
\hline & D10 & $26.4[25.5-30.6] \mathrm{Gy}$ & 18.8 [12.1-26.3] Gy & .009 \\
\hline & Dmax & 36.4 [36.1-37.6] Gy & 35.8 [33.1-37.1] Gy & .045 \\
\hline \multirow[t]{5}{*}{ Daily } & V35 & $0.9[0.7-1.5] \mathrm{cc}$ & $0.3[0.1-1.1] \mathrm{cc}$ & .025 \\
\hline & D2 & 33.5 [33.1-34.2] Gy & 28.7 [23.8-32.5] Gy & .007 \\
\hline & D5 & $31.0[29.5-33.0] \mathrm{Gy}$ & $24.3[19.5-30.7] \mathrm{Gy}$ & .008 \\
\hline & D10 & $27.1[24.5-31.1] \mathrm{Gy}$ & $19.9[15.3-27.4] \mathrm{Gy}$ & .014 \\
\hline & Dmax & $41.3[39.8-41.9] \mathrm{Gy}$ & 38.5 [36.4-41.9] Gy & .08 \\
\hline \multirow[t]{5}{*}{ Peak } & V35 & $1.4[1-2.3] \mathrm{cc}$ & $0.4[0.1-2.1] \mathrm{cc}$ & .04 \\
\hline & D2 & $34.5[33.8-36.0] \mathrm{Gy}$ & 31.7 [24.6-35.3] Gy & .04 \\
\hline & D5 & $32.3[31.2-34.5] \mathrm{Gy}$ & $25.2[20.6-31] \mathrm{Gy}$ & .005 \\
\hline & D10 & $28.7[26.5-31.5] \mathrm{Gy}$ & 20.9 [16.7-28.5] Gy & .01 \\
\hline & Dmax & $42.1[41.7-46.1] \mathrm{Gy}$ & $41.9[38.5-45.5] \mathrm{Gy}$ & .3 \\
\hline Stomach & & Acute pain (median [IQR]) & No acute pain (median [IQR]) & $p$ \\
\hline \multirow[t]{5}{*}{ Planned } & V35 & $0.1[0.0-0.4] \mathrm{cc}$ & $0.0[0.0-0.2] \mathrm{cc}$ & .11 \\
\hline & $\mathrm{D} 2$ & $31.9[27.3-32.9] \mathrm{Gy}$ & 25.8 [15.6-32.3] Gy & .06 \\
\hline & D5 & $29.3[24.6-30.9] \mathrm{Gy}$ & 20.5 [14.0-29.6] Gy & .023 \\
\hline & D10 & 25.6 [20.1-29] Gy & 16.3 [12.9-25.1]Gy & .012 \\
\hline & Dmax & $36.8[35.2-38.4] \mathrm{Gy}$ & 35.7 [26-36.8] Gy & .029 \\
\hline \multirow[t]{5}{*}{ Daily } & V35 & $0.3[0.0-0.9] \mathrm{cc}$ & $0.7[0.2-2.1] \mathrm{cc}$ & .17 \\
\hline & D2 & 32.5 [28.5-35] Gy & 27.9 [18.9-32.8] Gy & .016 \\
\hline & D5 & $29.0[25.2-31.7] \mathrm{Gy}$ & 22.7 [16.6-29] Gy & .012 \\
\hline & D10 & $26.0[21.1-28.5] \mathrm{Gy}$ & 18.7 [14.8-23.6] Gy & .005 \\
\hline & Dmax & 39.1 [30.4-40.5] Gy & 38.3 [36.6-42.3] Gy & .40 \\
\hline \multirow[t]{5}{*}{ Peak } & V35 & $1.8[0.4-3.2] \mathrm{cc}$ & $0.6[0.0-1.4] \mathrm{cc}$ & .08 \\
\hline & D2 & 34.7 [29.4-36.5] Gy & 31.0 [20.9-34.5] Gy & .034 \\
\hline & D5 & 32.1 [25.7-33.4]Gy & $26.2[17.3-31.1] \mathrm{Gy}$ & .01 \\
\hline & D10 & 28.9 [22.3-30.9]Gy & $20.9[15.4-25.8] \mathrm{Gy}$ & .05 \\
\hline & Dmax & $41.4[37.0-45.9] \mathrm{Gy}$ & $41.2[33.3-43.7] \mathrm{Gy}$ & .32 \\
\hline Bowel & & Acute diarrhea (median [IQR]) & No acute diarrhea (median [IQR]) & $p$ \\
\hline \multirow[t]{5}{*}{ Planned } & V35 & $0.0[0.0-0.0] \mathrm{cc}$ & $0.0[0.0-0.1] \mathrm{cc}$ & .21 \\
\hline & D2 & $28.0[22.7-31.2] \mathrm{Gy}$ & 18.8 [13.5-27.5] Gy & .09 \\
\hline & D5 & $25.2[21.3-27.9] \mathrm{Gy}$ & 16.7 [11.8-23.1] Gy & .07 \\
\hline & D10 & $23.1[20.0-24.9] \mathrm{Gy}$ & 15.1 [9.7-20.6] Gy & .034 \\
\hline & Dmax & $35.3[32.1-37.4] \mathrm{Gy}$ & 31.1 [20.9-36.2] Gy & .23 \\
\hline \multirow[t]{5}{*}{ Daily } & V35 & $0.7[0.1-1.5] \mathrm{cc}$ & $0.0[0.0-0.2] \mathrm{cc}$ & .13 \\
\hline & D2 & 29.8 [24.3-32.6] Gy & 16.9 [14.9-26.2] Gy & .07 \\
\hline & D5 & $27.0[22.3-29.5] \mathrm{Gy}$ & $19.0[12.5-22.2] \mathrm{Gy}$ & .038 \\
\hline & D10 & $24.2[20.4-26.4] \mathrm{Gy}$ & 14.6 [9.8-19.3] Gy & .027 \\
\hline & Dmax & 36.6 [31.4-39.9] Gy & 32.1 [22.2-38.7] Gy & .22 \\
\hline \multirow[t]{5}{*}{ Peak } & V35 & $1.2[0.3-3.4] \mathrm{cc}$ & $0.0[0.0-0.6] \subset c$ & .12 \\
\hline & D2 & 33.5 [26.8-37.6] Gy & 22.7 [16.8-28.8] Gy & .07 \\
\hline & D5 & $18.8[13.1-24.2] \mathrm{Gy}$ & 30.7 [24.2-33.8] Gy & .043 \\
\hline & D10 & 16.5 [10.9-21.1] Gy & 27.3 [21.6-29.7] Gy & .031 \\
\hline & Dmax & $41.8[35.0-45.0] \mathrm{Gy}$ & 35.9 [27.2-43.1] Gy & .35 \\
\hline
\end{tabular}

For extended analysis refer to Supplementary Table $2 \mathrm{~s}$. IQR: inter-quartile range.

Values reaching statistical significance $(p<0.05)$ are indicated in bold.
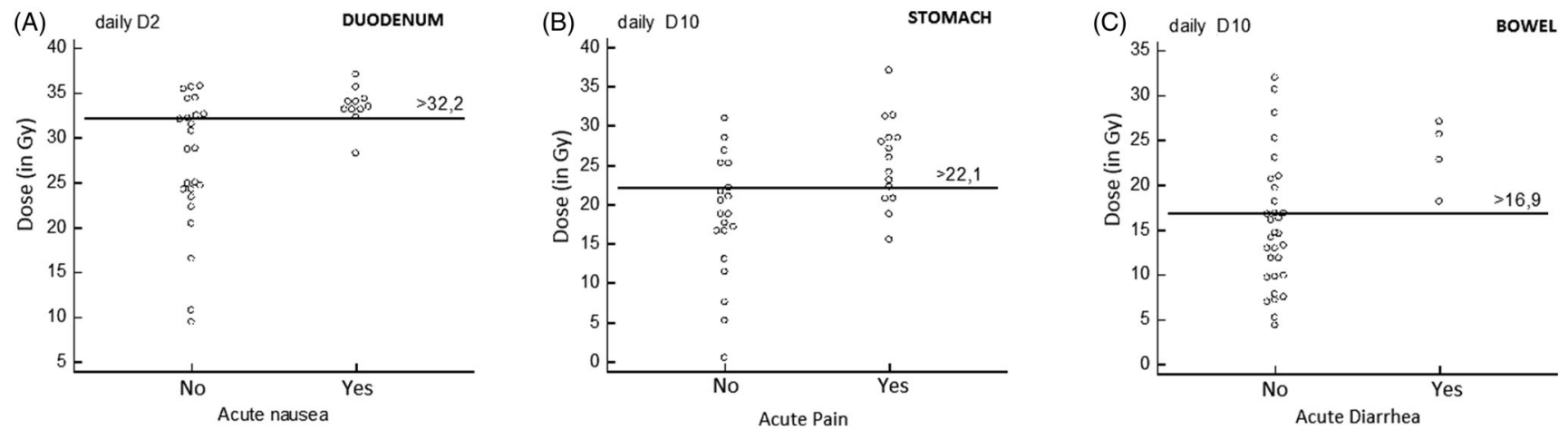

Figure 1. Dot chart summarizing relevant dose parameters for toxicity. Each patient is represented by a white dot; bar represent the value associated with Youden J. (A) Daily D2 to duodenum in patients with with (right) or without (left) acute nausea. (B) Daily D10 to stomach in patients with (right) or without (left) abdominal pain. (C) Daily D10 to bowel in patients with (right) or without (left) acute diarrhea. 
needed to confirm that these doses aresafe for late toxicity. More intensive dose regimens, delivering higher biological doses, may be of interest to increase response rate [10]: however due to proximity of radiosensitive critical structures, further dose escalation may expose to higher risk of adverse severe events. Moreover, initial experiences of daily imaging with cone-beam CT [22] and more recent trials implementing the use of high-definition imaging such as integrated MRI or helical CT suggest that large inter-fractional variations in shape and position occurs during the treatment course $[12,13,23]$, thus adding further uncertainty in determining the tolerance of OARs to radiation injury, since deformation and movement may allow critical structure to approach higher dose regions [12]. Hence, there is an urgent need to develop strategies to predict the risk of overirradiation of OARs and possibly compensate for uncertainties due to organ motion. In the present study dose estimates calculated from daily parameters (duodenum daily D2, stomach daily D10 and bowel daily D10), yielded superior accuracy in predicting acute toxicity. This finding has important implications, since daily assessment using high-quality diagnostic imaging provided more accurate prediction of clinical toxicities than exclusive simulation CT-based planning and may allow to prevent unintended dose constraints violation favored by organ motion. Daily dose recalculation at the means of helical CT or MRI may be applied to dose adaption based on daily assessment of anatomic variations [14,24]. Initial clinical application could be online evaluation of dose distribution to the organ at risk in order to aid clinical decision before treatment delivery in case of large inter-fractional variations that cannot be compensated by setup correction $[13,14]$.

Secondarily, development of daily adaptive protocols could be implemented: for example, selection of the plan (from a library of previously generated treatment plans or after full replanning) that, according to the anatomy of the day, allows the better therapeutic index between OAR sparing and target coverage [22].

Finally, mitigation of the risk of excessive OARs irradiation related to organ motion through daily imaging and dose adaptation may permit safe dose escalation to increase tumor control. In our cohort, dose thresholds for prediction of acute toxicity elaborated from daily assessment (i.e. duodenum daily D2 and stomach daily D10) compared favorably to their planned counterpart, resulting in concession of dose to the OARs of +1.7 and $+5.5 \mathrm{~Gy}$, respectively, thus improving the tradeoff between tumor coverage and organ sparing. These figures compare favorably with constraints enforced in trials of five-fraction pancreatic SBRT, in which D1cc < 33 Gy, D3cc < 20Gy, and D9cc < 15 Gy on stomach and small bowels were used [20]. Based on these results, an adaptive strategy using daily imaging could be proposed to manage uncertainty linked to organ motion and safely deliver higher doses of radiation without increasing the risk for acute toxicity [24].

There are several limitations in our work. Since LAPC-1 was not specifically designed to test the benefit of daily dose evaluation, according to study protocol only three daily CTs out of five fractions have been acquired to limit unnecessary irradiation and contrast product injection: future trials specifically designed should address this issue. Acute toxicity, consisting of moderate G1-2 complications, has been used as endpoint to test the predictive value of daily imaging, though constraints used for SBRT are mostly designed to prevent dose limiting, potentially life-threatening chronic side effects. Additional follow-up is needed to assess the onset of late toxicities before further dose escalation can be planned.

In conclusion, daily evaluation of dose to OAR using diagnostic-quality helical CT yields accurate prediction of acute toxicity following SBRT for inoperable pancreatic carcinoma. Its application in clinical practice may allow for implementation of image-guided dose adaptive strategies and safe dose escalation.

\section{Disclosure statement}

None of the authors have conflicts of interest to disclose.

\section{References}

[1] Strohl MP, Raigani S, Ammori JB, et al. Surgery for localized pancreatic cancer: the trend is not improving. Pancreas. 2016;45(5): 687-693.

[2] Suker M, Beumer BR, Sadot E, et al. FOLFIRINOX for locally advanced pancreatic cancer: a systematic review and patient-level meta-analysis. Lancet Oncol. 2016;17(6):801-810.

[3] Faris JE, Blaszkowsky LS, McDermott S, et al. FOLFIRINOX in locally advanced pancreatic cancer: the Massachusetts General Hospital Cancer Center experience. Oncologist. 2013;18(5): 543-548.

[4] Sadot E, Doussot A, O'Reilly EM, et al. FOLFIRINOX induction therapy for stage 3 pancreatic adenocarcinoma. Ann Surg Oncol. 2015;22(11):3512-3521.

[5] Katz MH, Shi Q, Ahmad SA, et al. Preoperative modified FOLFIRINOX treatment followed by capecitabine-based chemoradiation for borderline resectable pancreatic cancer: alliance for clinical trials in oncology trial A021101. JAMA Surg. 2016;151(8): e161137.

[6] Zhong J, Patel K, Switchenko J, et al. Outcomes for patients with locally advanced pancreatic adenocarcinoma treated with stereotactic body radiation therapy versus conventionally fractionated radiation. Cancer. 2017;123(18):3486-3493.

[7] Petrelli F, Comito T, Ghidini A, et al. Stereotactic body radiation therapy for locally advanced pancreatic cancer: a systematic review and pooled analysis of 19 trials. Int J Radiat Oncol Biol Phys. 2017;97(2):313-322.

[8] Katz MHG, Ou FS, Herman JM, for the Alliance for Clinical Trials on Oncology, et al. Alliance for clinical trials in oncology (ALLIANCE) trial A021501: preoperative extended chemotherapy vs. chemotherapy plus hypofractionated radiation therapy for borderline resectable adenocarcinoma of the head of the pancreas. BMC Cancer. 2017;17(1):505.

[9] Chuong MD, Springett GM, Freilich JM, et al. Stereotactic body radiation therapy for locally advanced and borderline resectable pancreatic cancer is effective and well tolerated. Int J Radiat Oncol Biol Phys. 2013;86(3):516-522.

[10] Rudra S, Jiang N, Rosenberg SA, et al. Using adaptive magnetic resonance image-guided radiation therapy for treatment of inoperable pancreatic cancer. Cancer Med. 2019;8(5):2123-2132.

[11] Liu F, Erickson B, Peng C, et al. Characterization and management of interfractional anatomic changes for pancreatic cancer radiotherapy. Int J Radiat Oncol Biol Phys. 2012;83(3):e423-9. 
[12] Loi M, Magallon-Baro A, Suker M, et al. Pancreatic cancer treated with SBRT: effect of anatomical interfraction variations on dose to organs at risk. Radiother Oncol. 2019; 134:67-73.

[13] El-Bared N, Portelance L, Spieler BO, et al. Dosimetric benefits and practical pitfalls of daily online adaptive mri-guided stereotactic radiation therapy for pancreatic cancer. Pract Radiat Oncol. 2019;9(1):e46-e54.

[14] Papalazarou C, Klop GJ, Milder MTW, et al. CyberKnife with integrated CT-on-rails: System description and first clinical application for pancreas SBRT. Med Phys. 2017; 44(9):4816-4827.

[15] Youden WJ. Index for rating diagnostic tests. Cancer. 1950;3(1): 32-35.

[16] Kelly P, Das P, Pinnix CC, et al. Duodenal toxicity after fractionated chemoradiation for unresectable pancreatic cancer. Int J Radiat Oncol Biol Phys. 2013;85(3):e143-9.

[17] Kavanagh BD, Pan CC, Dawson LA, et al. Radiation dose-volume effects in the stomach and small bowel. Int J Radiat Oncol Biol Phys. 2010;76(3):S101-S7.

[18] Nakamura A, Shibuya K, Matsuo Y, et al. Analysis of dosimetric parameters associated with acute gastrointestinal toxicity and upper gastrointestinal bleeding in locally advanced pancreatic cancer patients treated with gemcitabine-based concurrent chemoradiotherapy. Int J Radiat Oncol Biol Phys. 2012;84(2):369-375.
[19] Schellenberg D, Goodman KA, Lee F, et al. Gemcitabine chemotherapy and single-fraction stereotactic body radiotherapy for locally advanced pancreatic cancer. Int J Radiat Oncol Biol Phys. 2008;72(3):678-686.

[20] Herman JM, Chang DT, Goodman KA, et al. Phase 2 multi-institutional trial evaluating gemcitabine and stereotactic body radiotherapy for patients with locally advanced unresectable pancreatic adenocarcinoma. Cancer. 2015;121(7):1128-1137.

[21] Mellon EA, Hoffe SE, Springett GM, et al. Long-term outcomes of induction chemotherapy and neoadjuvant stereotactic body radiotherapy for borderline resectable and locally advanced pancreatic adenocarcinoma. Acta Oncol. 2015;54(7):979-985.

[22] Li Y, Hoisak JD, Li N, et al. Dosimetric benefit of adaptive re-planning in pancreatic cancer stereotactic body radiotherapy. Med Dosim. 2015;40(4):318-324.

[23] Magallon-Baro A, Loi M, Milder M, et al. Modeling daily changes in organ-at-risk anatomy in a cohort of pancreatic cancer patients. Radiother Oncol. 2019;134:127-134.

[24] Bruynzeel AME, Lagerwaard FJ. The role of biological dose-escalation for pancreatic cancer. Clin Transl Radiat Oncol. 2019;18: 128-130. 